\title{
Anesthesia management for cesarean section 10 years after heart transplantation: a case report
}

\author{
Xiaofei Qi ${ }^{1}$, Xiaolei Wang ${ }^{2}$, Xiaolei Huang ${ }^{1}$, Chenhong Wang ${ }^{3}$, Yin Gu and Yuantao $\mathrm{Li}^{1 *}$
}

\begin{abstract}
Introduction: Pregnancy after organ transplantation is becoming increasingly common. However, reports of the anesthesia for such patients are rare. Heart transplant recipients are always accompanied with pathophysiological changes and present anesthesiologists with challenge.
\end{abstract}

Case description: We reported a case of anesthesia management of gravida undergoing cesarean section 10 years after cardiac transplantation. We used two points spinal and epidural anesthesia, combined with phenylephrine throughout the surgery. The course was absolutely successful and both mother and baby got good results.

Discussion and evaluation: Physiology of heart transplant recipients and key points of anesthesia management were discussed.

Conclusions: Spinal anesthesia can be performed in heart transplant recipients, however, we have to think twice before anesthesia for this kind of patients.

Keywords: Anesthesia, Cardiac transplantation, Cesarean section, Pregnancy

\section{Introduction}

For severe end-stage heart disease, cardiac transplantation is a life-saving procedure for those are refractory to medical therapies. Nowadays, the overall survival of recipients has increased to about $90 \%$ at 1 year and more than $75 \%$ at 7 years post transplantation (Taylor et al. 2007). In these heart transplanted recipients, women constitute one-third and about $20 \%$ of them are in reproductive age (Alston et al. 2001).

Cardiac-transplanted patients present anesthesiologists with challenging problems related to the function of the denervated heart and their complex drug therapies. If combined with pregnancy, changes accompanied with pregnancy should be taken into account, and the condition will be more complicated.

\footnotetext{
${ }^{*}$ Correspondence: yuantaoli6788@163.com

1 Department of Anesthesiology, Shenzhen Maternity and Child Healthcare Hospital, Southern Medical University, Shenzhen 518028 Guangdong, China

Full list of author information is available at the end of the article
}

We reported the successful outcome of anesthesia for a pregnancy undergoing cesarean section 10 years after cardiac transplantation for a dilated cardiomyopathy. We used intrathecal anesthesia, combined with vasoconstrictor throughout the surgery. The course was uneventful and hemodynamic stable.

\section{Case description}

A 33-year-old pregnant woman was admitted to hospital on 19th, March, 2015 with gestation of 34 weeks and 3 days. She underwent orthotopic cardiac transplantation in September 2005 for a dilated cardiomyopathy. During remaining 10 years she was treated with immunosuppressor tacrolimus and mycophenolate on schedule and no rejection episode was noted. Seven months ago she found she was pregnant and stopped mycophenolate according to the doctor's advice. During pregnancy, antenatal cares were performed timely and no obstetrical complications were found. After discussion of obstetricians, cardiologists, neonatologists and anesthesiologists, 
cesarean section was decided to perform on gestation of 35 weeks for her history of heart transplantation.

\section{Preoperative evaluation}

The parturient was 35 weeks gestation and $56 \mathrm{~kg}$ on the day of surgery (24th, March, 2015). The patient's general condition was good and cardiac function classification was stage one. ECG showed sinus tachycardia: 110 beats per minute. Cardiac ultrasound showed left ventricular wall thickening and ascending aortic dilatation. Laboratory tests: $\mathrm{Hb} 95 \mathrm{~g} / \mathrm{l}$. WBC $13.2 \times 10^{9} / \mathrm{l}$. Coagulation function, liver and kidney function were normal.

\section{Anesthesia procedure}

The patient fasted overnight and no preoperative medication was administered. Tacrolimus was treated orally $1.5 \mathrm{mg} / 12 \mathrm{~h}$ until morning of surgery. On arrival in the operating room, pulse oxygen saturation, electrocardiogram, and non-invasive blood pressure were monitored, and the baseline values were recorded. Oxygen $(5 \mathrm{l} / \mathrm{min})$ by facemask was given until delivery. An intravenous catheter was placed and the patient was preloaded with Lactated Ringer's Solution (12-15 ml/kg) before induction of spinal anesthesia. Left radial artery was punctured and catheter was inserted to measure direct blood pressure. Deep venous puncture was not performed.

Two points of combined spinal and epidural anesthesia (CSEA) was performed with the patient in the lateral decubitus position. Firstly at L2-3 intervertebral space epidural catheter was placed $3 \mathrm{~cm}$ cephalic through epidural needle. Then at L3-4 intervertebral space a 25G spinal Quincke needle was introduced to subarachnoid space, after free flow of cerebral spinal fluid (CSF), $0.5 \%$ ropivacaine $10 \mathrm{mg}$ was injected at a rate of $0.1 \mathrm{ml} / \mathrm{s}$. The patient was immediately placed in the supine position with uterus leftward.

At the same time when anesthesia performed, intravenous phenylephrine was pumping continuously at rate of $0.1 \mu \mathrm{g} / \mathrm{kg}$.min to prevent hypotension. After injection of intrathecal medication, the rate of phenylephrine was adjusted between 0.1 and $0.3 \mu \mathrm{g} / \mathrm{kg}$ min according to patient's hemodynamic condition.

When satisfactory anesthesia level (T6) was achieved, surgery began. Five minutes later, a male infant was delivered, weighing $2150 \mathrm{~g}$ with Apgar score of 10 at 1 and 5 min after delivery. Immediately after baby was born, oxytocin 10 units was given intramuscular in the uterus and 10 units intravenous dripping with added to $500 \mathrm{ml}$ Lactated Ringer's Solution. The surgery lasted for $35 \mathrm{~min}$ and the course was uneventful, the parturient complained no discomfort. During the surgery, blood pressure maintained at $108-122 / 65-82 \mathrm{mmHg}$, and heart rate at $80-108$ beats/min. The total volume of infusion fluid was $1500 \mathrm{ml}$, blood loss was $200 \mathrm{ml}$, urine was $100 \mathrm{ml}$. When the operation was over, epidural morphine $2 \mathrm{mg}$ was given via epidural catheter and patient controlled intravenous analgesia (PCIA) pump was treated. The pump was total $100 \mathrm{ml}$ contained $1 \mu \mathrm{g} / \mathrm{ml}$ sufentanil with background flow $2 \mathrm{ml} / \mathrm{h}$, bolus $4 \mathrm{ml}$, locked time $30 \mathrm{~min}$.

She was taken care in intensive care unit postoperaton. $3.5 \mathrm{~h}$ later, anesthesia was completely subsided. Tacrolimus was treated orally $1.5 \mathrm{mg} / 12 \mathrm{~h}$ continually. Breast feeding was not allowed for the risk of immunosuppressant to baby. Six days after delivery she got good recovery with no complications and was discharged from hospital together with her infant. Follow up was carried out 5 months and no episode was found.

\section{Discussion and evaluation}

For heart transplanted recipients undergoing non-cardiac surgeries, we should recognize the physiology of the transplanted heart, pharmacologic effects of immunosuppressive medications and complications accompanied by heart transplantation. For pregnant women, obstetrical conditions also should be considered. We should take care of the patients from preoperative period, intraoperative period, and postoperative period.

\section{Physiology of transplanted heart and preoperative evaluation and preparation}

The transplanted heart is denervated. The remaining atrial cuff of recipient is innervated but hemodynamic unimportant. The donor atrium is denervated but is responsible for the electrophysiological responses of the transplanted heart. It retains its intrinsic control mechanisms which include: a normal Frank-Starling effect, normal impulse formation and conductivity, intact a and $\beta$ receptors responding to circulating catecholamines (Blasco et al. 2009). At rest, the heart rate is faster than normal at about 90-100 beats per minute because lack of vagal tone (Ramakrishna et al. 2009).

Including the function of transplanted heart, we also have to notice the complications following heart transplantation and the influence of anti-rejection drugs in the heart transplanted recipients. Nearly $75 \%$ of post-transplant recipients develop mild to moderate hypertension as a result of immunosuppressor therapy (O'Boyle et al. 2010). Because cardiac responsiveness during exercise is dependent on circulating catecholamines, beta blockers are best avoided after heart transplantation (Blasco et al. 2009). The denervated heart is vulnerable to an accelerated process of coronary atherosclerosis. Allograft coronary atherosclerotic disease is present in $10-20 \%$ of patients 1 year after transplantation and in near $50 \%$ by 5 years (Ng and Cassorla 2007). Even in angiographically normal coronary arteries, coronary luminal narrowing 
may develop insidiously. The lack of afferent innervation renders episodes of myocardial ischaemia silent in these patients. Therefore, diagnostic ECG is essential in the perioperative period. If coagulation function is abnormal, intravertebral anesthesia should be avoided. Many immune inhibitors, nonstemidial anti-inflammatory drugs are nephrotoxic drugs, so anesthetics that are excreted mainly by renal clearance should be avoided. Immunosuppressant caused infection remains a major cause of death (Aguero et al. 2008; Van de Beek et al. 2008), thus aseptic technique should be paramount. Invasive monitoring techniques and all forms of instrumentation should be handled with sterile manipulation.

Pregnancy is associated with significant hemodynamic demands. Blood volume increases by $40 \%$ and cardiac output by $30 \%$. The transplanted heart is denervated and so responds to these demands with adaptive mechanisms: an increase in central venous pressure and preload leads to an increase in stroke volume. Circulating catecholamines allow further increases in cardiac output by increasing heart rate and contractility. If pre-pregnancy cardiac function is normal, the transplanted heart is generally able to adjust to these demands (Wu et al. 2007). In reported cases of pregnancies following heart transplantation, outcomes of pregnancy have been good with no recurrence of cardiac dysfunction in the transplanted heart (Armenti et al. 2008; Humphreys et al. 2012; Kalinka et al. 2014). However, the incidence of maternal complications is increased in heart transplant recipients (Miniero et al. 2004; Sibanda et al. 2007). Hypertension is a significant problem both prior to and during pregnancy (Zurbano et al. 2012; Armenti et al. 2004; Coscia et al. 2010) and it requires meticulous control. The incidence of preeclampsia is approximately $20 \%$ (Zurbano et al. 2012; Armenti et al. 2004; Coscia et al. 2010).

\section{Anesthesia management}

Cesarean section is performed in about $30 \%$ of heart transplanted recipients (Cowan et al. 2012; Wielgos et al. 2009). No matter what anesthesia method to perform, we should maintain hemodynamic stable and protect cardiac function, and keep mother and baby safe. Both general and intravertebral anesthesia were successfully performed in heart transplanted patients (Valerio et al. 2014; Allard et al. 2004). But for pregnant women, the better anesthesia choice is intravertebral anesthesia which produces less impact on baby compared with general anesthesia. This patient is with good cardiac function, normal coagulation function and no other serious complications, so spinal anesthesia was performed to prevent impact of general anesthetics to baby. For the post heart transplanted patients, several points we should notice:
Firstly, appropriate anesthesia level must be controlled. For too high anesthesia level will inhibit sympathetic nerve, dilate vessel which is unfavorable for transplanted heart. The same, too low anesthesia level is not enough for the surgery, and pain will increase oxygen consumption of myocardium. We controlled anesthesia level at T6, and got satisfactory effect and hemodynamic stable.

Secondly, appropriate fluid infusion. The normal heart increases its cardiac output via neural stimuli leading to increases in heart rate and contractility (Schwaiblmair et al. 1999), while the denervated heart lacks the ability to respond acutely to hypovolaemia or hypotension with reflex tachycardia, and dependent upon venous return with an initial increase in left ventricular end-diastolic volume (Blasco et al. 2009; Swami et al. 2011), which mediates an increase in stroke volume and ejection fraction by means of the Frank-Starling mechanism. Adequate preload must be ascertained preoperatively and intravascular volume status maintained intraoperatively. However, too much fluid is also not beneficial for denervated heart, for risks to increase heart load and lead to heart failure. We preloaded $12-15 \mathrm{ml} / \mathrm{kg}$ Ringer lactate solution before anesthesia to resist vasodilation caused by anesthesia.

Thirdly, vasoconstrictor phenylephrine was treated intravenously to maintain intravascular volume and keep hemodynamic stable. Phenylephrine is $\alpha$ receptor agonist. As intraspinal anesthesia dilates vessel and relatively decrease blood volume, so phenylephrine is helpful to maintain intravascular volume and keep hemodynamic stable, and doesn't affect myocardial contractivity. The transplanted heart is more sensitive to drugs directly acts on heart such as adrenaline, norepinephrine, isoprensline than those indirectly drugs as ephedrine, metaradrine. The heart rate shows no response to drugs like atropine, neostigmine, phenylephrine, but will respond to isoproterenol, ephedrine, dopamine.

Fourth, antiseptic measures. Maternal infection is of significant concern, although it is relatively rare in practice. It is recommended that all procedures are performed with strict asepsis and antibiotic prophylaxis be used for all operative and instrumental deliveries. We sterilized carefully before spinal anesthesia procedures, and antibiotics were used throughout the surgery. Invasive central venous pressure (CVP) was not used in this case because of the patient's preoperative stability, minimal surgical risk, and the low possibility of massive fluid infusion.

\section{Postoperative care}

The parturient was taken good care in ICU. Immunosuppressant drugs tacrolimus was continued to use postoperatively as Knight and Morris suggested (Knight and Morris 2007). Analgesia must be good enough to avoid 
increasing oxygen consumption of myocardium. In addition, intravenous fluids must be well maintained, and urine output monitored.

\section{Conclusions}

For anesthesia in gravidas following heart transplantation, we should recognize the physiology of the transplanted heart, pharmacologic effects of immunosuppressive medications, obstetrical condition of patients. In addition, understand the importance of preload dependence, proper administration of direct vasoactive drugs if needed, and aware infectious risk. Take care of the patient from preoperative period, intraoperative period, and postoperative period. The most important is to make cardiac function normal, hemodynamic stable, enable mother and baby safe.

\section{Abbreviations}

CSEA: combined spinal and epidural anesthesia; CSF: cerebral spinal fluid; PCIA: patient controlled intravenous analgesia; CVP: central venous pressure; ICU: intensive care unit.

\section{Authors' contributions}

$X Q$ : First author who grafted the article; YL: Corresponding author who was in charge of the clinical job and chief responsible for revising the manuscript; $\mathrm{XH}$ and $\mathrm{XW}$ : Chief anesthesiologists of the surgery; CW: Chief operater of the surgery; YG: Revise the manuscript and English polishing. All authors read and approved the final manuscript.

\section{Author details}

1 Department of Anesthesiology, Shenzhen Maternity and Child Healthcare Hospital, Southern Medical University, Shenzhen 518028, Guangdong, China. 2 Department of Anesthesiology, Sun Yat-Sen Cardiovascular Hospital of Shenzhen, Shenzhen 518028, Guangdong, China. ${ }^{3}$ Department of Gynecology and Obstetrics, Shenzhen Maternity and Child Healthcare Hospital, Southern Medical University, Shenzhen 518028, Guangdong, China.

\section{Competing interests}

All authors declare that they have no competing interests.

\section{Consent for publication}

Our work has notified the patient and written consent was obtained from the patient.

\section{Ethics approval and consent to participate}

Ethics approval and consent have obtained from ethics committee of Shenzhen Maternal and Child Healthcare Hospital.

Received: 23 January 2016 Accepted: 28 June 2016

Published online: 07 July 2016

\section{References}

Aguero J, Almenar L, Martinez-Dolz L et al (2008) Influence of immunosuppressive regimens on short-term morbidity and mortality in heart transplantation. Clin Transpl 22:98-106
Allard R, Hatzakorzian R, Deschamps A (2004) Decreased heart rate and blood pressure in a recent cardiac transplant patient after spinal anesthesia. Can J Anesth 51:829-833

Alston PK, Kuller JA, McMahon MJ (2001) Pregnancy in transplant recipients. Obstet Gynecol Surv 56:289-295

Armenti VT, Radomski JS, Moritz MJ et al. (2004) Report from the National Transplantation Pregnancy Registry NTPR: outcomes of pregnancy after transplantation. Clin Transplant 103-114

Armenti VT, Constantinescu S, Moritz MJ et al (2008) Pregnancy after transplantation. Transplant Rev (Orlando, Fla) 22(4):223-240

Blasco LM, Parameshwar J, Vuylsteke A (2009) Anaesthesia for noncardiac surgery in the heart transplant recipient. Curr Opin Anesthesiol 22(1):109-113

Coscia LA, Constantinescu S, Moritz MJ et al (2010). Report from the National Transplantation Pregnancy Registry (NTPR): outcomes of pregnancy after transplantation. Clin Transplant 65-85

Cowan SW, Davison JM, Doria C et al (2012) Pregnancy after cardiac transplantation. Cardiol Clin 30(3):441-452

Humphreys RA, Wong HH, Milner R, Matsuda-Abedini M (2012) Pregnancy outcomes among solid organ transplant recipients in British Columbia. J Obstet Gynaecol Can JOGC 34(5):416-424

Kalinka J, Szubert M, Zdziennicki A et al (2014) A second delivery after heart transplantation - a case study. Kardiochir Torakochirurgia Pol 11(3):339-342

Knight SR, Morris PJ (2007) The clinical benefits of cyclosporine C2-level monitoring: a systematic review. Transplantation 83(12):1525-1535

Miniero R, Tardivo I, Centofanti P et al (2004) Pregnancy in heart transplant recipients. J Heart Lung Transplant 23:898-901

$\mathrm{Ng}$ V, Cassorla L (2007) Cardiac transplant recipient undergoing noncardiac surgery. In: Bready LL, Noorily NH, Dillman D (eds) Decision making in anesthesiology: an algorithmic approach, 4th edn. Mosby Elsevier, Philadelphia, pp 468-471

O'Boyle PJ, Smith JD, Danskine AJ et al (2010) De novo HLA sensitization and antibody mediated rejection following pregnancy in a heart transplant recipient. Am J Transpl 10(1):180-183

Ramakrishna H, Jaroszewski DE, Arabia FA (2009) Adult cardiac transplantation: a review of perioperative management Part-I. Ann Card Anaesth 12(1):71-78

Schwaiblmair M, von Scheidt W, Uberfuhr P et al (1999) Functional significance of cardiac reinnervation in heart transplant recipients. J Heart Lung Transplant 18:838-845

Sibanda N, Briggs JD, Davison JM, Johnson RJ, Rudge CJ (2007) Pregnancy after organ transplantation: a report from the UK transplant pregnancy registry. Transplantation 83:1301-1307

Swami AC, Kumar A, Rupal S, Lata S (2011) Anaesthesia for non-cardiac surgery in a cardiac transplant recipient. Indian J Anaesth 55(4):405-407

Taylor DO, Brown RN, Jessup ML et al (2007) Progress in heart transplantation: riskier patients yet better outcomes: a 15 year multi-institutional study. J Heart Lung Transplant 26:S61

Valerio R Jr, Durra O, Gold ME (2014) Anesthetic considerations for an adult heart transplant recipient undergoing noncardiac surgery: a case report. AANA J 82(4):293-299

Van de Beek D, Kremers WK, del Pozo JL et al (2008) Effects of infectious diseases on outcome after heart transplant. Mayo ClinProc 83:304-308

Wielgos M, Pietrzak B, Bobrowska K et al (2009) Pregnancy after organ transplantation. Neuro Endocrinol Lett 30(1):6-10

Wu DW, Wilt J, Restaino S (2007) Pregnancy after thoracic organ transplantation. Semin Perinatol 31(6):354-362

Zurbano F, Lorez F, Fornet I et al (2012) Maternity and lung transplantation: cases in Spain. Arch Bronconeumol 48(10):379-381 